\title{
A new material for the icy Galilean moons: The structure of sulfuric acid hexahydrate
}

\author{
H. E. Maynard-Casely, ${ }^{1}$ K. S. Wallwork, ${ }^{1}$ and M. Avdeev ${ }^{2}$ \\ Received 12 February 2013; revised 9 July 2013; accepted 9 August 2013; published 20 September 2013.
}

[1] This study presents a newly identified water-rich crystalline form of sulfuric acid hydrate, $\mathrm{H}_{2} \mathrm{SO}_{4} \cdot 6 \mathrm{H}_{2} \mathrm{O}$, a hexahydrate. The method of formation of this material suggests that sulfuric acid hexahydrate (SAHx) could be an abundant material on the surface of Jupiter's Galilean ice moons, Europa, Ganymede, and Callisto. The structure of SAHx was determined by the combined use of synchrotron X-ray and neutron powder diffraction data. The structural arrangement of SAHx exhibits the same water layer topology that has been determined for sulfuric acid octahydrate (SAO), but differs in the stacking of this water layer and the interlayer species. SAHx is observed to form over a large range of solution compositions and displays stability over the temperature range 80 to $190 \mathrm{~K}$.

Citation: Maynard-Casely, H. E., K. S. Wallwork, and M. Avdeev (2013), A new material for the icy Galilean moons: The structure of sulfuric acid hexahydrate, J. Geophys. Res. Planets, 118, 1895-1902, doi:10.1002/jgre.20124.

\section{Introduction}

[2] Alongside the wealth of geological features that the Galilean moons exhibit [Prockter et al., 2010] is the variety of newly identified ice minerals that have formed these landscapes. Aside from Io, where the mineralogy is dominated by silicates, the rest of the Galilean moons' surfaces are dominated by ice and ice hydrates [Dalton et al., 2010]. There is strong evidence that sulfuric acid hydrates are prevalent on the surface of Europa [Carlson et al., 1999, 2002] and that these materials could also be present on Jupiter's largest moon Ganymede, the target for the Europan Space Agency's JUICE mission [Boutonnet and Schoenmaekers, 2012]. The case for the presence of sulfuric acid hydrates on Europa is justified by the abundance and transport of sulfate ions from Io [Cheng, 1984], and from the fitting of spectral data collected by the Galileo spacecraft's Near Infra-Red Mapping Spectrometer (NIMS) [Carlson et al., 1999]. Despite detailed mapping of the extent of sulfuric acid hydrate across Europa's surface, predicting up to $90 \%$ sulfuric acid hydrate fraction in some regions [Carlson et al., 2005], very few models of surface landforms have taken account of the presence of these materials.

[3] This is perhaps because it not fully understood which phases of sulfuric acid hydrate would exist in the water-rich environments of the icy Galilean moons. It is consequently impossible to assign crystallographic structures and commensurably

Additional supporting information may be found in the online version of this article.

${ }^{1}$ Australian Synchrotron, Clayton, Victoria, Australia.

${ }^{2}$ Bragg Institute, Australian Nuclear Science and Technology Organisation, Kirrawee DC, New South Wales, Australia.

Corresponding author: H. E. Maynard-Casely, Bragg Institute, Australian Nuclear Science and Technology Organisation, Locked Bag 2001, Kirrawee DC, New South Wales, 2232, Australia. (helen.maynard-casely@ansto.gov.au)

(C)2013. American Geophysical Union. All Rights Reserved. 2169-9097/13/10.1002/jgre.20124 difficult to attribute phases to spectroscopic data with any certainty. These two questions are addressed by this study. Determination of which sulfuric acid hydrates are present as a function of solution composition, coupled with detailed knowledge of these materials response to temperature, enables the extraction of information such as density, water content and other physical properties. A full understanding of these hydrate's crystal structures, including the positions of each of the hydrogen atoms, provides molecular speciation information, which can then assist in the assignment of features observed in spectroscopy data.

[4] The variety of hydrates formed within the sulfuric acidwater binary system have been studied for a century [Gable et al., 1950; Giran, 1913; Hornung et al., 1956; Hulsmann, 1934; Pickering, 1890; Rubin and Giauque, 1952]. These previous works have explored a complex phase diagram and established that there are six crystalline forms in the system; a monohydrate, a dihydrate, a trihydrate, a tetrahydrate, a hemitriskaidekahydrate and an octahydrate. Of these known forms, the water-rich sulfuric acid hydrates are thought to be most relevant to the Galilean satellites [Fortes and Choukroun, 2010]; that is, sulfuric acid octahydrate (SAO) and hemitriskaidekahydrate (SAH).

[5] Described herein is the discovery of a previously unknown water-rich sulfuric acid hydrate, sulfuric acid hexahydrate (SAHx), which is structurally distinct from each of the hydrates previously observed in this binary system. A full crystal structure determination of this new phase is presented, derived from synchrotron X-ray powder diffraction and neutron powder diffraction data. The thermal expansion of SAHx has also been charted, between 80 and $190 \mathrm{~K}$, and its expansivity over this range has been established from these data.

\section{Methods and Measurements}

[6] This study investigated the frozen products of 11 different aqueous solutions of sulfuric acid. Nine of these solutions, prepared for the in situ synchrotron X-ray powder 
diffraction experiment, were made with hydrogenous material $\left(\mathrm{H}_{2} \mathrm{SO}_{4}\right.$ and $\left.\mathrm{H}_{2} \mathrm{O}\right)$ from sulfuric acid obtained from Sigma Aldrich (99.999\% pure) and distilled water. These solutions were prepared by weight with a $0.001 \mathrm{~g}$ balance. The first eight sample solutions were $8.8 \mathrm{wt} \%, 16.9 \mathrm{wt} \%, 24.4 \mathrm{wt} \%, 31.4 \mathrm{wt} \%$, $37.9 w t^{0} \%, 40.5 w t \%, 43.9 w t \%$, and $55.5 \mathrm{wt} \%$ aqueous sulfuric acid. A sample made from a $47.6 \mathrm{wt} \%$ aqueous sulfuric acid solution was studied during a follow-up synchrotron experiment; this stoichiometric mixture of sulfuric acid hexahydrate provided further confirmation of the new phase, and the variable temperature data were used to chart thermal expansion. A further two samples were prepared for in situ neutron diffraction experiments which were conducted with a similar methodology; these samples were prepared with the deuterated equivalent reagents, $\mathrm{D}_{2} \mathrm{SO}_{4}$ and $\mathrm{D}_{2} \mathrm{O}$. The reagents were obtained from Sigma Aldrich. The samples were prepared as $37.0 \mathrm{wt} \%$ and $47.0 \mathrm{wt} \% \mathrm{D}_{2} \mathrm{SO}_{4}$ solutions. Nonstoichiometric compositions, such as these promote the crystallization of additional Ice Ih and sulfuric acid containing products, thereby improving the signal of the powder diffraction data.

[7] In order to optimize the crystallization of the samples for study by diffraction, the propensity for the samples to form a glass phase on quenching [Kunzler and Giauque, 1952; Fortes et al., 2006] was exploited. Each sample was rapidly cooled to $\sim 100 \mathrm{~K}$ and then warmed at a rate of $6 \mathrm{~K}$ per minute to around $170-180 \mathrm{~K}$. At this latter point, each of the samples was observed to crystallize, and the completeness of crystallization was monitored by the diffraction method in use (X-ray or neutron). Once fully crystalline, each sample was cooled at the rate of $6 \mathrm{~K}$ per minute to the base temperature of the apparatus used $(80 \mathrm{~K}$ with a nitrogen gas flow cryostream for the X-ray measurements, and $3 \mathrm{~K}$ with a liquid helium cryostat for the neutron measurements). The samples were cooled to reduce the thermal motion of the sample and, in the case of the neutron diffraction measurements, to aid in the search for deuterium (hydrogen) positions.

[8] The synchrotron X-ray diffraction experiment was undertaken at the powder diffraction beamline at the Australian Synchrotron [Wallwork et al., 2007]. The nine sample solutions were each loaded into $0.3 \mathrm{~mm}$ diameter borosilicate capillaries, along with strands of silica wool to seed crystallization. Once mounted onto the beamline, the temperature of the sample was controlled by an Oxford Cryosystems Cryostream Plus [Cosier and Glazer, 1986]. Although the cryostream nozzle is positioned as close as practicable to the sample, the cryostream's thermocouple is not at the sample position and therefore the temperature is calibrated in a way that reflects the sample temperature. To achieve the calibration, the melting point of a number of well-characterized materials is monitored by diffraction and adjustments between observed and device-reported temperature are made, where necessary. Calibration samples (and melting points) relevant to the temperature range of this study are: $\operatorname{argon}(88 \mathrm{~K})$, isoprene $(127 \mathrm{~K})$, ethanol $(159 \mathrm{~K})$, and 2-propanol $(184 \mathrm{~K})$. From this calibration, it is shown that over the temperature range studied in this paper $(80-180 \mathrm{~K})$ the difference between the calibrated temperature and that reported by the cryostream is less than $1 \mathrm{~K}$. X-ray diffraction patterns were collected with the MYTEHN-II strip detector [Bergamaschi et al., 2010]; the initial experiment saw eight of these acquired with an X-ray wavelength of $0.9895(1) \AA$, and the later experiment examined an additional sample (47.6 wt $\%$ solution) at
$0.9996(1) \AA$. The wavelength was determined by Rietveld refinement to data acquired from the NIST standard $\mathrm{LaB}_{6}$ number $660 \mathrm{~b}$.

[9] The neutron diffraction experiment was undertaken at the Echidna beamline (high-resolution powder diffraction) at the OPAL reactor at the Australian Nuclear Science and Technology Organisation (ANSTO) [Liss et al., 2006]. Data collected from two compositions, $37.5 \mathrm{wt} \%$ and $47.0 \mathrm{wt} \%$, are described here. Each solution was transferred into a $5 \mathrm{~mm}$ diameter quartz capillary along with $\sim 0.8 \mathrm{~g}$ of silica wool to seed crystallization. The quartz capillaries containing the silica wool and sample solutions were then each placed inside a $9 \mathrm{~mm}$ vanadium can for neutron diffraction. As with the synchrotron X-ray diffraction experiment, data were collected from the samples in situ. During the neutron diffraction experiment, the sample temperature was controlled with a liquid helium cryostat (temperature range $1.8-300 \mathrm{~K})$. To ensure that the full sample volume was quenched, the sample canisters were plunged into liquid nitrogen, and the temperature monitored throughout, via the cryostat's temperature controller. The cryostat stick and sample canister were then loaded into a precooled cryostat at $100 \mathrm{~K}$; again, the temperature was monitored during this process and was shown not to exceed $120 \mathrm{~K}$. Once the sample was in position on the diffractometer, the temperature of the sample was varied using the same regime as described for the X-ray experiment. Neutron diffraction patterns were collected at a wavelength of $2.4395(5) \AA$.

[10] Indexing of the diffraction patterns was undertaken with the TOPASv4.1 refinement package [Coelho, 2008], and subsequent refinement of the unit cell and crystal structures (both X-ray and neutron) was undertaken in both TOPASv4.1 and GSAS refinement packages [Larson and Dreele, 1994]. Determination of the "heavy atom" (sulfur and oxygen) positions within the unit cell was undertaken with Parallel Tempering (PT) methods within version 1.19 of the Free Objects for Crystallography (FOX) software [Favre-Nicolin and Cerny, 2002]. Verification of the completeness of the structure proposed herein was conducted using PLATON [Spek, 2009].

\section{Results}

[11] A series of sulfuric acid solutions, ranging in composition from $8.8 \mathrm{wt} \%$ to $55.5 \mathrm{wt} \%$ sulfuric acid, were studied initially by synchrotron X-ray powder diffraction. The temperature regime of sample preparation was designed to model a possible pathway for sulfuric acid hydrate production on the surface of the Galilean ice moons. Independent of whether the sulfate would have arrived at the surface exogenically [Carlson et al., 1999] or endogenically [Dalton et al., 2010], the crystalline products (thought to exist $\sim 1 \mathrm{~mm}$ under the surface [Dalton et al., 2010]) are expected to have formed from the warming of an amorphous glass phase.

[12] Upon crystallization of solutions with a weight percent of sulfuric acid of $40.5 \mathrm{wt} \%$ or below, three distinct crystalline phases were always observed in the pattern, in varying proportions. Two were recognized as sulfuric acid octahydrate (SAO) and Ice Ih. The diffraction peaks from the unidentified material could not be matched to that of any of the known phases of sulfuric acid hydrate or ice. The repeated presence of this observed, yet uncharacterized, 

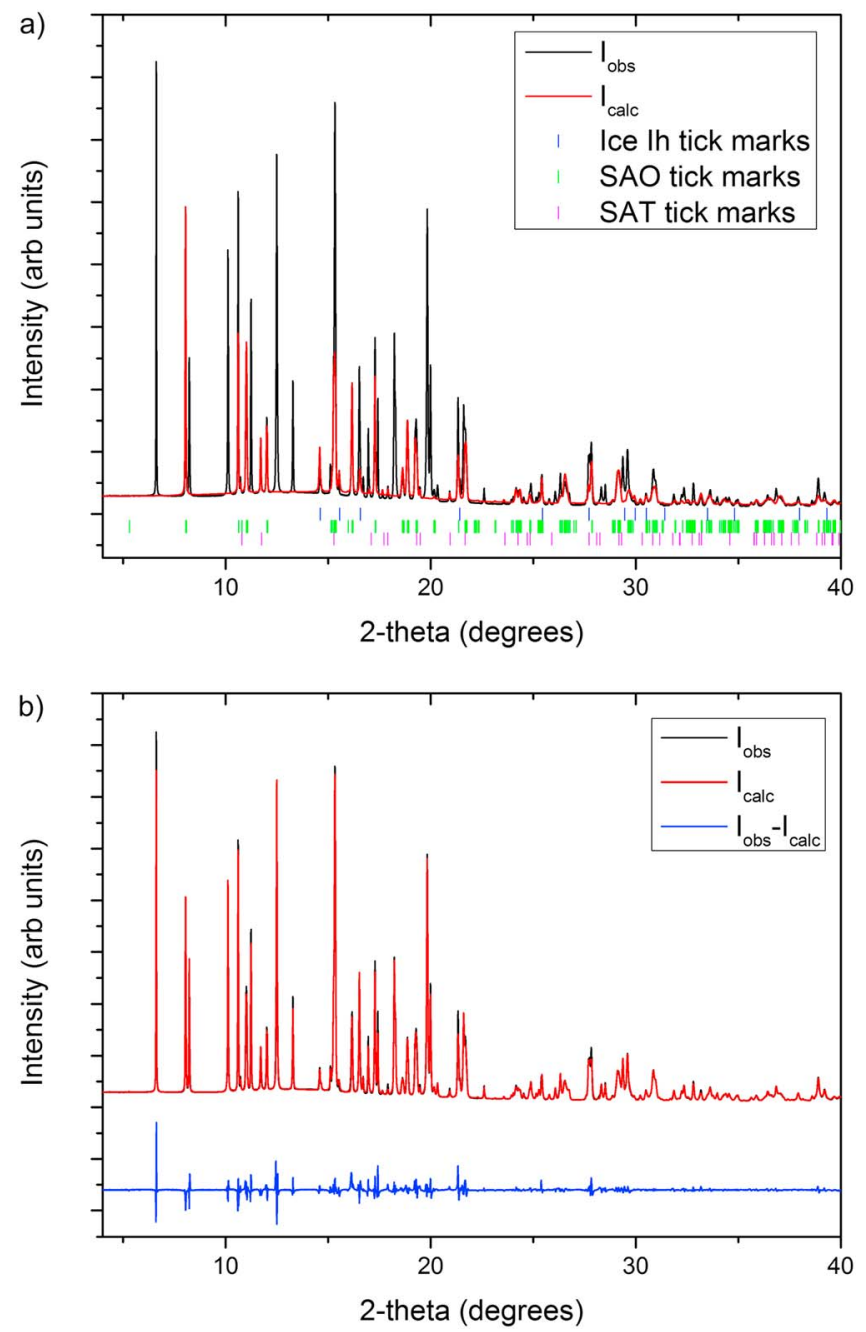

Figure 1. Synchrotron $X$-ray powder diffraction pattern obtained from a $44.0 \mathrm{wt} \%$ sulfuric acid solution, each of the plots is linear with the difference curves presented on the same scale. (a) The pattern with only the crystal structures of Ice Ih, sulfuric acid octahydrate (SAO), and sulfuric acid tetrahydrate (SAT) fitted to it, clearly leaving a significant amount of the pattern unaccounted for. These remaining peaks were used in the indexing of the new form, sulfuric acid hexahydrate (SAHx). (b) The same pattern, but with a Pawley fit to the orthorhombic SAHx cell included.

pattern across all of the samples studied has now been shown to be a previously undiscovered form of sulfuric acid hydrate - described below. Where the composition of the prepared solution was greater than $40.5 \mathrm{wt} \%$ sulfuric acid, four phases were observed, again in varying proportions. As in the more dilute solutions, three of these phases were Ice Ih, SAO, and the unknown phase. The additional phase in these solutions was identified as sulfuric acid tetrahydrate (SAT).

[13] Using the pattern presented in Figure 1, and omitting the peaks from Ice Ih, SAO, and SAT, the new sulfuric acid hydrate form was indexed to an orthorhombic unit cell with $a=7.4154(1), b=7.5156(1), c=17.0864(2)$, volume 952.25 (2) $\AA^{3}$. A Pawley fit of this cell yielded a fit to the orthorhombic cell with an overall pattern $\mathrm{R}_{\mathrm{wp}}$ of $7.04 \%$.

[14] Once the unit cell of the unknown phase was established and fit to the diffraction data, the space group symmetry was determined for this material. Because of the complex nature of the diffraction data, determining systematic reflection absences of the unknown phase to ascertain its unit cell symmetry was challenging. From Pawley refinement, the pattern of the unknown hydrate was seen to conform to the constraints of the $P 2_{1} 2_{1} 2_{1}$ space group; hence, structure solution was undertaken with this symmetry (a fuller account of this justification is given in the supporting information).

[15] Building on the information of unit cell and symmetry, the next stage was to try and find the atomic positions within the unknown phase. The low X-ray scattering of the hydrogen atoms in the sample required that only the "heavy atom" (sulfur and oxygen) positions could be ascertained from the X-ray data. Determination of the heavy atom positions within the unknown phase was undertaken by the optimization of structural models with parallel tempering against the diffraction data collected from the $44.0 \mathrm{wt} \%$ solution at $80 \mathrm{~K}$; this sample displayed the highest proportion of the unknown phase in the diffraction pattern.

[16] In order to establish the unit cell contents, a comparison of the unit cell size, the symmetry, and the possible molecular components was made. It was expected that the unknown phase would display a density comparable to that of the octahydrate at $80 \mathrm{~K}\left(1344 \mathrm{kgm}^{-3}\right)$ [Maynard-Casely 


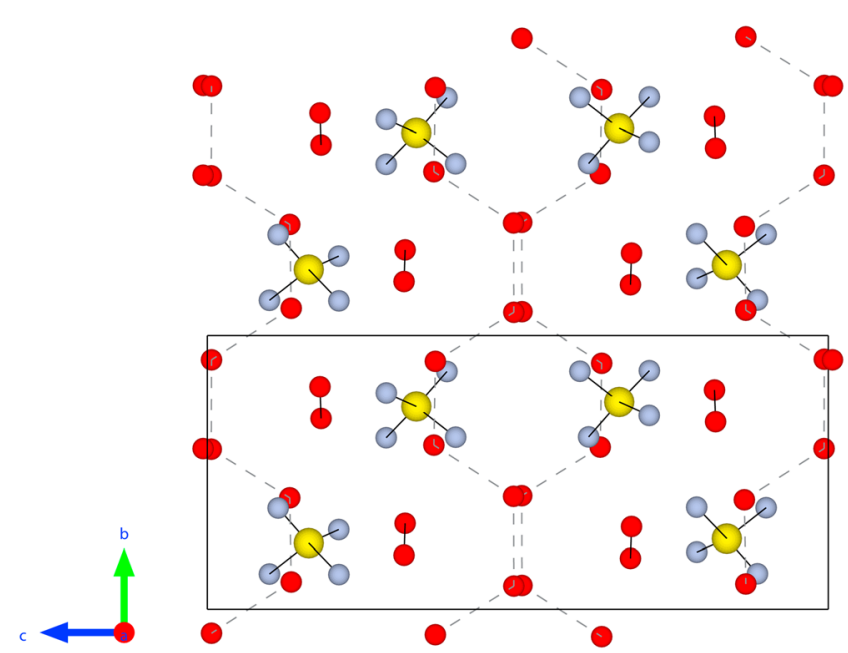

Figure 2. Illustration of the heavy atom structure of sulfuric acid hexahydrate. The arrangement of sulfate ions and oxygen atoms in the sulfuric acid hexahydrate structure determined by this study along the $a$ axis. The sulfate ions are shown with the sulfur as yellow atoms and their associated oxygen atoms in light blue. The red oxygen atoms represent the water network in the structure. Dashed lines have been drawn between water oxygen atoms where the distance between them is less than $3 \AA$.

et al., 2012], hemitriskaidekahydrate at $184 \mathrm{~K}\left(1538.8 \mathrm{kgm}^{-3}\right)$ [Mootz and Merschenz-Quack, 1987], or tetrahydrate at $83 \mathrm{~K}$ $\left(1569 \mathrm{kgm}^{-3}\right)$ [Kjallman and Olovsson, 1972]. In addition, the cell contents needed to comply with the symmetry of the Orthorhombic space group. Given these constraints, the feasible composition was deemed to be either sulfuric acid hexahydrate $\left(\mathrm{H}_{2} \mathrm{SO}_{4} \bullet 6 \mathrm{H}_{2} \mathrm{O}\right)$ or septahydrate $\left(\mathrm{H}_{2} \mathrm{SO}_{4} \bullet 7 \mathrm{H}_{2} \mathrm{O}\right)$; respectively, they have densities of $1424 \mathrm{kgm}^{-3}$ and $1550 \mathrm{kgm}^{-3}$ at $80 \mathrm{~K}$ (derived from the unit cell volume of $952.23 \AA^{3}$ ). Both of these options were pursued for structure elucidation using the data collected from $44.0 \mathrm{wt} \%$ solution, at

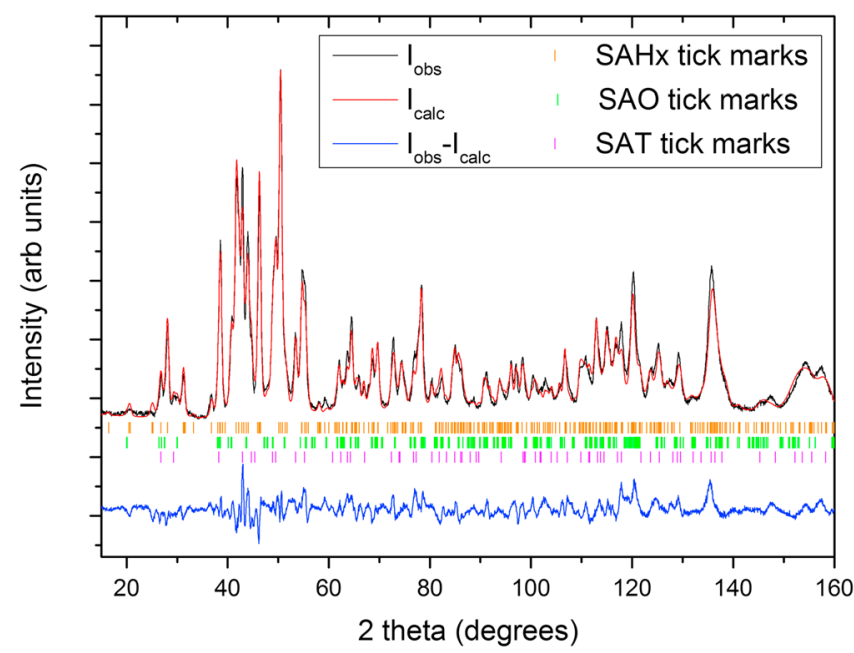

Figure 3. Fit of the SAHx structure to neutron diffraction data, formed from a loaded solution of $47.0 \mathrm{wt} \% \mathrm{D}_{2} \mathrm{SO}_{4}$ in $\mathrm{D}_{2} \mathrm{O}$ and collected at $3 \mathrm{~K}$. Fitted against this pattern are SAHx, SAT, and SAO; the details of which are given in the supporting information - Table S2.
80 K. Structure elucidation was carried out with Parallel Tempering (PT) methods within the Free Objects for Crystallography (FOX) software [Favre-Nicolin and Cerny, 2002] with two potential models. The first calculation optimized a model comprising of initially randomized positions of one sulfate molecule and six oxygen atoms (hexahydrate) and the other using a similarly initially randomized model with one sulfate molecule and seven oxygen atoms (septahydrate).

[17] The unknown phase was solved from an optimization of $P 2{ }_{1} 2_{1} 2_{1}$ unit cell with one sulfate ion and six oxygen atoms input. From this structure solution, it was revealed that the unknown phase is sulfuric acid hexahydrate (SAHx). This conclusion was reached from the fact that the hexahydrate calculation reaches a satisfactory minimum with a viable structure but the septahydrate calculation did not. This solution was further verified with PLATON [Spek, 2009], which discerned no voids for additional water or sulfate molecules. Figure 1 yielded an $\mathrm{R}_{\mathrm{Bragg}}$ of $4.90 \%$ with an overall fit of the pattern (with SAO, SAT, and Ice Ih also fitted) of $\mathrm{R}_{\mathrm{wp}} 10.89 \%$. The heavy atom structure of SAHx is presented in Figure 2 and shows a remarkable similarity to the heavy atom structure of sulfuric acid octahydrate (SAO), which will be discussed later.

[18] To locate the hydrogen atom positions in the crystal structure of sulfuric acid hexahydrate (SAHx), neutron powder diffraction data of this phase were collected. In this case, the incoherent nature of neutron scattering from hydrogen atoms requires that hydrogen is replaced with deuterium; thus, mixtures of $\mathrm{D}_{2} \mathrm{SO}_{4}$ and $\mathrm{D}_{2} \mathrm{O}$ were investigated. Location of the deuterium (hydrogen) atoms provides key information on the ionic species that exist within the structure, a property that has a strong bearing upon the understanding of a material's spectral properties.

[19] As in the X-ray study, the neutron diffraction study shows that SAHx forms in the presence of SAO, SAT, and Ice Ih. The stoichiometry for the deuterated phase, $\mathrm{D}_{2} \mathrm{SO}_{4} \cdot 6 \mathrm{D}_{2} \mathrm{O}$, is $44.4 \mathrm{wt} \%$. However, it was possible to eliminate the crystallization of ice by preparation of a slightly higher concentration of sulfuric acid solution; $47.0 \mathrm{wt} \%$, resulted in a

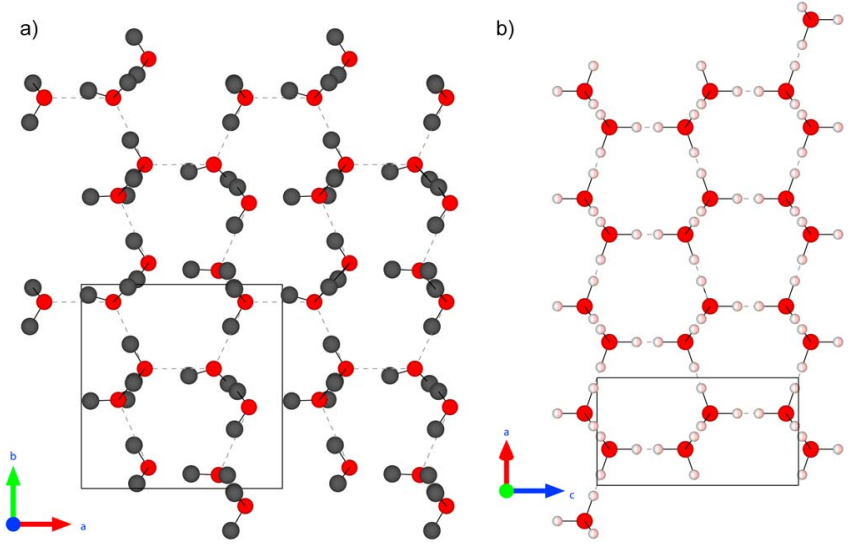

Figure 4. (a) Illustration of the water network in SAHx, compared with (b) a view of the [011] sheet in Ice Ih structure (interpreted from [Peterson and Levy, 1957]). The oxygen atoms are depicted in red, deuterium atoms in dark grey, and hydrogen atoms in pink. 

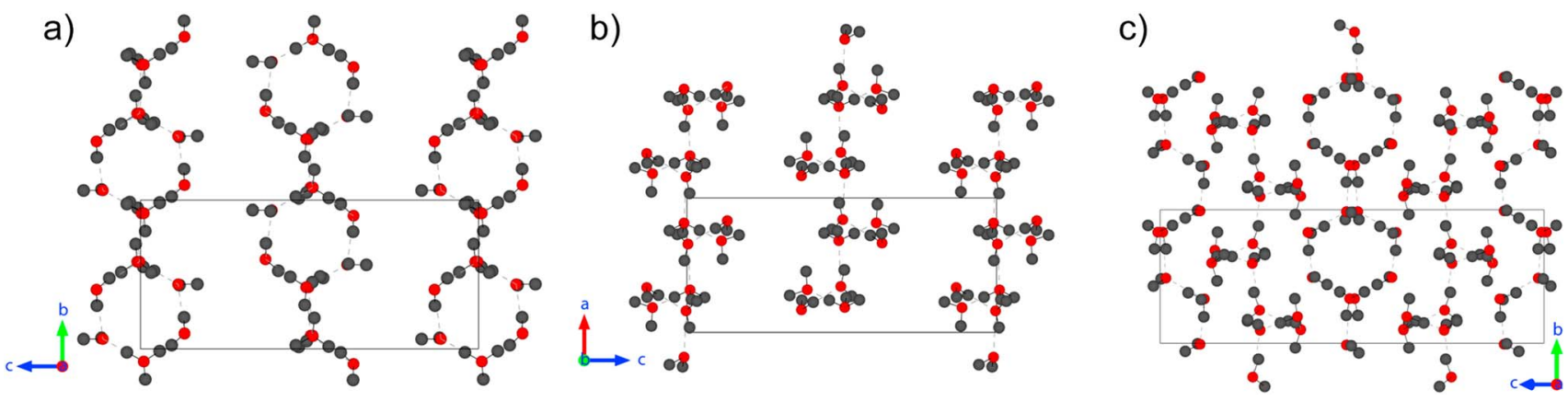

Figure 5. Illustration of the $\mathrm{D}_{3} \mathrm{O}^{+} / \mathrm{D}_{2} \mathrm{O}$ network in SAHx, viewed (a) down the $a$ axis and (b) down the $b$ axis, in comparison to (c) that within SAO. The red dots depict oxygen atoms and deuterium atoms are represented by dark grey dots. Hydrogen bonds are indicated with dashed lines.

simpler sample comprising SAO, SAT, and SAHx. Data were collected from this sample at $3 \mathrm{~K}$ and these data were used for determining the deuterium (hydrogen) atom positions within SAHx. Using these data, the deuterium atom positions within SAO were also determined, as explained below and in the supporting information.

[20] Deuterium positions were located initially with Fourier difference maps, generated from the neutron diffraction data combined with the heavy atom structure determined from the X-ray powder diffraction study of SAHx (this work); the heavy atom structure of SAO was determined in earlier work [Maynard-Casely et al., 2012]. Positive electron density residuals identified by these maps were used to locate the presence of deuterium atoms in the structure. The positions of the deuterium atoms were then refined with constraint to the bonded oxygen atom in a rigid body arrangement (detailed in supporting information). The best fit to these data, shown in Figure 3, was obtained for both $\mathrm{SAO}$ and $\mathrm{SAHx}$ where the water layers consisted of $\mathrm{D}_{3} \mathrm{O}^{+}$and $\mathrm{D}_{2} \mathrm{O}$ molecules.

[21] This work presents that the structure of SAHx consists of $\mathrm{D}_{3} \mathrm{O}^{+}$and $\mathrm{D}_{2} \mathrm{O}$ layers, which sandwich a layer of $\mathrm{D}_{5} \mathrm{O}_{2}{ }^{+}$and $\mathrm{SO}_{4}{ }^{2-}$ ions, giving the structural formula $\left[\left(\mathrm{SO}_{4}\right)^{2-}\left(\mathrm{D}_{5} \mathrm{O}_{2}\right)^{+}\right]\left[\left(\mathrm{D}_{3} \mathrm{O}\right)^{+}\left(\mathrm{D}_{2} \mathrm{O}\right)_{3}\right]$. The topology of the $\mathrm{D}_{3} \mathrm{O}^{+} / \mathrm{D}_{2} \mathrm{O}$ water layers is of a single sheet of the Ice Ih structure cleaved along the [011] plane and then buckled by the presence of the $\mathrm{D}_{5} \mathrm{O}_{2}{ }^{+}$and $\mathrm{SO}_{4}{ }^{2-}$ ions, as shown in Figure 4. The departure from the Ice Ih topology is because the deuterium bond bridging between the [011] planes is broken by a buckling of the chain. This buckling leaves a deuterium bonded "ring" containing 10 oxygen centers (composed of $\mathrm{D}_{3} \mathrm{O}^{+}$and $\mathrm{D}_{2} \mathrm{O}$ species). It is two adjacent rings like this that gives a larger space than in the Ice Ih network to accommodate for the $\mathrm{D}_{5} \mathrm{O}_{2}{ }^{+}$ and $\mathrm{SO}_{4}{ }^{2-}$ species that are sandwiched between the layers. The layers are stacked in an A B motif, with layer B being a translation of layer $\mathrm{A}$ by half a unit cell in the $b$ axis direction.

[22] The $\mathrm{D}_{3} \mathrm{O}^{+} / \mathrm{D}_{2} \mathrm{O}$ water layers in SAHx are almost identical to those identified in SAO; the contrast in their structures arises from differences in the stacking of these layers, resulting in the higher water content of the latter. Figure 5 illustrates the difference in this layering; the SAO structure is composed of alternating $\mathrm{D}_{3} \mathrm{O}^{+} / \mathrm{D}_{2} \mathrm{O}$ water layers $\mathrm{A}$ and $\mathrm{B}$, with layer $\mathrm{B}$ being a rotation of $\mathrm{A} 90^{\circ}$ about the $b$ or $c$ axis. (Figure $5 \mathrm{c}$ ). This results in smaller cavities between the layers, which can only accommodate one $\mathrm{SO}_{4}{ }^{2-}$ ion, as opposed to the $\mathrm{D}_{5} \mathrm{O}_{2}{ }^{+}$ and $\mathrm{SO}_{4}{ }^{2-}$ ion pair that is contained between the layers in SAHx (Figures 5a and 5b).
[23] Sulfuric acid hexahydrate was observed to form in eight of the nine compositions studied by X-ray diffraction, ranging from $8.8 \mathrm{wt} \%$ to $43.9 \mathrm{wt} \%$ sulfuric acid, as shown in Figure 6. Although SAHx is prevalent across the compositions studied, the maximum relative proportion observed is relatively low, compared to the other hydrate phases, thereby suggesting that this phase has lower stability than other members of its family.

[24] To further probe the stability of SAHx, a variable temperature experiment was conducted on a stoichiometric (1:6) solution $47.6 \mathrm{wt} \% \mathrm{H}_{2} \mathrm{SO}_{4}$ solution with in situ synchrotron $\mathrm{X}$-ray powder diffraction. For this experiment, the sample was frozen and crystallized by the same procedure described for the first eight samples. This sample crystallized into a mixture of SAHx, SAO, and SAT in the relative proportions given in Figure 6, 58.8\% SAHx, 22.9\% SAO, and $18.3 \%$ SAT. Following collection of a powder diffraction pattern at $80 \mathrm{~K}$, the sample temperature was stepped in $5 \mathrm{~K}$ intervals to a maximum temperature of $190 \mathrm{~K}$, with data acquired at each temperature point. Rietveld refinement to these data

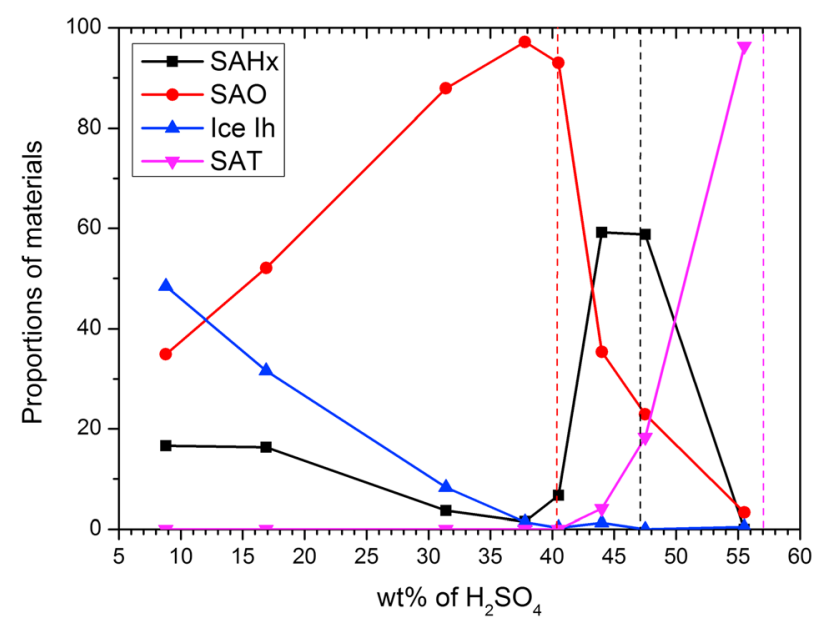

Figure 6. The variation in the relative proportions of each hydrate obtained from semiquantitative analysis across the $\left(\mathrm{H}_{2} \mathrm{O}\right.$ based) solutions studied at $80 \mathrm{~K}$. The errors in these determined proportions are contained within the symbols used to plot each point. The results from the $24.4 \mathrm{wt} \%$ solution sample have been omitted as a loss of temperature control during the experiment leads to a change in the experimental conditions. 


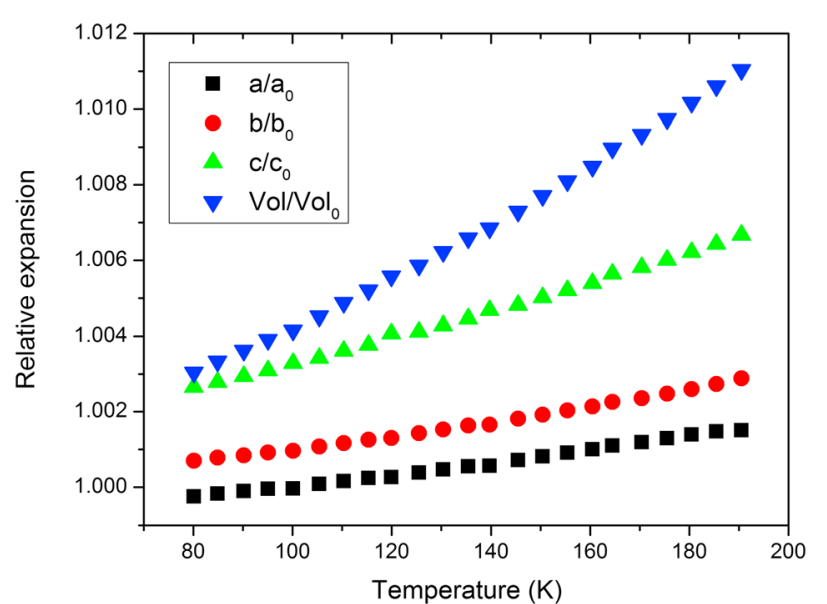

Figure 7. The relative thermal expansion of sulfuric acid hexahydrate between 80 and $190 \mathrm{~K}$. The initial values $\left(\mathrm{V}_{0}\right.$, $\mathrm{a}_{0}, \mathrm{~b}_{0}$, and $\mathrm{c}_{0}$ ) were determined from third-order polynomial fits to the variation of data with temperature to be $\mathrm{V}_{0}=950.6(8)$, $\mathrm{a}_{0}=7.421(3), \mathrm{b}_{0}=7.510(2)$, and $\mathrm{c}_{0}=17.06(1)$. Errors in the data points are contained within the symbols used to plot these data.

was undertaken, and each data set was fit with the crystal structures of SAHx, SAO, and SAT. The resulting variation in lattice parameters and volume, relative to the parameters observed at $80 \mathrm{~K}$, for SAHx is presented in Figure 7.

[25] The expansion of SAHx unit cell volume with temperature has been modelled with a fourth-order polynomial of $\mathrm{AT}^{3}+\mathrm{BT}^{2}+\mathrm{CT}+\mathrm{V}_{0}$ where $\mathrm{A}$ is $-1.9 \times 10^{-7} \mathrm{~K}^{-3}( \pm 3 \times$ $\left.10^{-7}\right), \mathrm{B}$ is $2.2 \times 10^{-4} \mathrm{~K}^{-2}\left( \pm 1 \times 10^{-4}\right), \mathrm{C}$ is $1.9 \times 10^{-2} \mathrm{~K}^{-1}$ $\left( \pm 1.5 \times 10^{-2}\right)$, and $V_{0}$ is $950.8 \AA^{3}( \pm 0.6)$. Figure 7 shows that, like the expansion of SAO [Maynard-Casely et al., 2012], the thermal expansion of SAHx is anisotropic with more expansion occurring along the $c$ axis. This is because of the relatively limited extend of hydrogen bonding along the $c$ axis; Figures 5a and $5 \mathrm{~b}$ illustrate the fact that the majority of the hydrogen bonding in SAHx is confined to the $a b$ plane.

\section{Discussion}

[26] This study set out to understand the identity of waterrich sulfuric acid hydrate(s) that could feasibly form on icy Galilean moons. Herein we have outlined the experimental evidence that sulfuric acid octahydrate (SAO) and the newly discovered sulfuric acid hexahydrate (SAHx) are the two crystalline forms of sulfuric acid hydrate that would most likely occur on the water-rich environments of the Europan surface and possibly subsurface. In the light of these results, sulfuric acid hexahydrate should be considered in any spectroscopic analysis conducted on data from the Galileo spacecraft's NIMS instrument and knowledge of the crystal structures of these materials, presented here, are invaluable input information into the library of materials used to fit data acquired by future remote sensing missions to these icy moons. Determination of crystal structure is also vital in the exploration of a material's stability in terms of temperature and pressure, and for the extraction of thermo-elastic properties.

[27] This research has demonstrated that a broad range ice and hydrate phases, and combinations thereof may form, thereby highlighting the importance of a complete understanding of such materials in order to be able hypothesize on the formation of planetary surface features.

\subsection{Thermal Stability of Sulfuric Acid Hydrate Phases}

[28] The discovery of a new water-rich sulfuric acid hydrate has further diversified the phase diagram of the sulfuric acid-water binary system. There are now four known waterrich phases (i.e., phases are observed to occur in solutions of $50 \mathrm{wt} \%$ sulfuric acid and below), a tetrahydrate, a hexahydrate, and hemitriskaidekahydrate, and an octahydrate. It is intriguing that we did not at any point in our studies observe the formation of sulfuric acid hemitriskaidekahydrate (SAH), despite observation of this phase by other researchers [Fortes et al., 2006]. The qualitative evidence presented in this paper does not exclude the possibility that SAH could form on the surfaces or within the subsurface of the Galilean moons. The differences in the sulfuric acid hydrates formed in the Fortes et al. study and this work suggest that the formation of these phases is very sensitive to parameters like quenching rate and temperature variation. As such, the thermodynamics of the phase diagram warrant further investigation. Knowledge of all the possible hydrate phases and understanding of their crystal structures are essential steps to undertaking such studies. Indeed, the richness of the data acquired thus far suggests that there is still much to learn about the relative thermal stability of the sulfuric acid hydrates. A detailed understanding of the formation and molecular speciation of all four hydrates (SAHx, SAH, SAO, and SAT), from crystallographic data, could lead to the ability to map the distribution of each these hydrate phases separately and serve as markers for different geophysical conditions across the surface of the icy Galilean moons.

\subsection{Thermal Expansivity of Sulfuric Acid Hexahydrate}

[29] The surfaces of the icy Galilean moons may exhibit features caused by the presence of these sulfuric hydrates mixed with Ice Ih. In this case, it may be possible to comment on the likely nature of such features, with knowledge of the

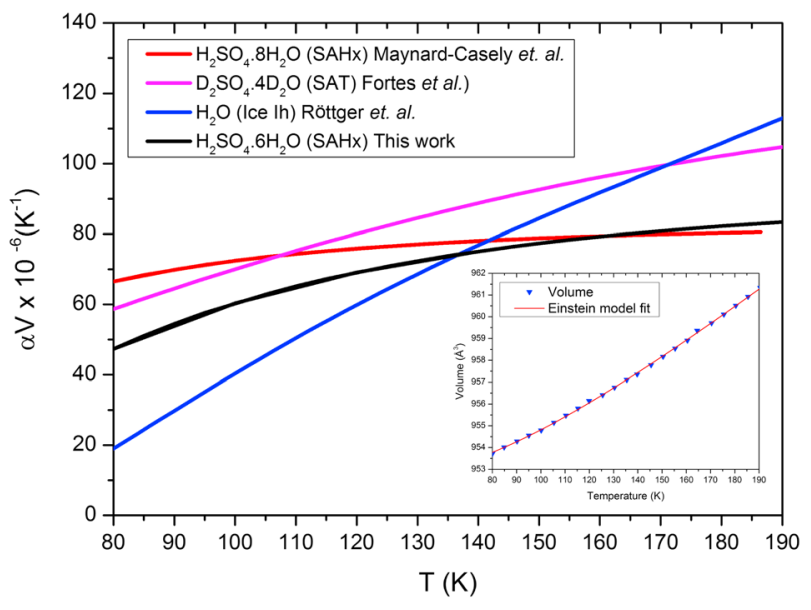

Figure 8. Thermal expansivities of the water-rich sulfuric acid hydrates (derived from this work as well as work by Maynard-Casely et al. [2012] and Fortes et al. [2008b]) compared to that of Ice Ih [Rottger et al., 2012]. Inset is the fit of the Einstein model to the volume data. The error in these data points is contained with the symbols used to plot them. 
thermal expansion behavior of the phases involved. We have extracted the thermal expansivity of SAHx, between 80 and $190 \mathrm{~K}$; a temperature range which is consistent with expectations of the environment. Thermal expansivity has been determined by fitting an Einstein oscillator model to the volume change over this temperature range. The use of this model has been discussed previously [Fortes et al., 2008a] and has been employed to characterize similar samples where no heat capacity data have been available [Brand et al., 2009; Maynard-Casely et al., 2012]. The variation in unit cell volume was fit with the same expression as used in Maynard-Casely et al., and the fit to the Einstein oscillator model is presented in the inset of Figure 8.

[30] Figure 8 illustrates similar thermal expansion behavior between SAHx and SAO, confirming that similarities between their crystal structure has a bearing on the physical properties of these materials. The thermal expansivity for both of these materials exceeds that of ice between 80 and $135 \mathrm{~K}$. The additional expansivity that the sulfuric acid hydrates exhibit can be attributed to the layered nature of the crystal structures, compared to the three-dimensional interconnectivity observed in Ice Ih. Given that temperatures on Europa range from 25 to $132 \mathrm{~K}$ [Spencer et al., 1999], any change in the temperature of a crust composed of a mixture of sulfuric acid hydrates and ice would be subjected to local variations in strain as a result of this effect. This could result in small-scale features and textures able to form on the surface independent of any large-scale geological processes.

\subsection{Spectral Properties of Sulfuric Acid Hexahydrate and Sulfuric Acid Octahydrate}

[31] This work has highlighted the fact that analysis of the NIMS has been incomplete because of the omission of sulfuric acid hexahydrate from the spectral library. As with the different forms of magnesium sulfate hydrate (epsomite and meridianiite), which are considered in the library, it seems that different forms of sulfuric acid hydrate (hexahydrate, octahydrate, etc.,) also need to be considered. It is expected that the different hydration states and the variety of molecular species that make up the structures of sulfuric acid hydrates will impact the spectral properties, making them distinct also.

[32] The combination of neutron and X-ray diffraction has allowed a comprehensive insight into these structures, providing evidence (from determination of perdeuterated equivalents) that the speciation within $\mathrm{SAO}$ is $\left[\left(\mathrm{SO}_{4}\right)^{2-}\right]$ $\left[\left(\mathrm{H}_{3} \mathrm{O}\right)^{+}\left(\mathrm{H}_{2} \mathrm{O}\right)_{3}\right]_{2}$ and the newly discovered and characterized SAHx is composed of $\left[\left(\mathrm{SO}_{4}\right)^{2-}\left(\mathrm{H}_{5} \mathrm{O}_{2}\right)^{+}\right]\left[\left(\mathrm{H}_{3} \mathrm{O}\right)^{+}\left(\mathrm{H}_{2} \mathrm{O}\right)_{3}\right]$. The speciation within the SAO structure, determined by diffraction, is contrary to earlier reports of the composition, namely $\left(\mathrm{SO}_{4}\right)^{2-}\left(\mathrm{H}_{5} \mathrm{O}_{2}\right)_{2}^{+}\left(\mathrm{H}_{2} \mathrm{O}\right)_{4}$, which has been used in previous calculations of the sulfuric acid hydrate distribution across Europa [Carlson et al., 2005]. The diffraction investigation of SAO, like a previous determination of the speciation of sulfuric acid hemitriskaidekahydrate [Fortes et al., 2006], has shown the presence of $\mathrm{H}_{3} \mathrm{O}^{+}$and $\mathrm{H}_{2} \mathrm{O}$ species, contrary to predictions from DFT calculations [Hirsch and Ojamäe, 2004]. This serves to illustrate the difficulty in obtaining the minimum energy conformations in such lowsymmetry structures. The present findings agree with the suggestion that the distorted water bands on Europa are due to $\mathrm{H}_{3} \mathrm{O}^{+}$species [Dalton et al., 2010]; hydronium ions have been identified by this study to occur in the structures of both SAO and SAHx. Although SAH, SAHx, and SAO each contain $\mathrm{H}_{3} \mathrm{O}^{+}$and $\mathrm{H}_{2} \mathrm{O}$ species, only $\mathrm{SAHx}$ contains the $\mathrm{H}_{5} \mathrm{O}_{2}{ }^{+}$ species within its crystal structure. The unique presence of $\mathrm{H}_{5} \mathrm{O}_{2}{ }^{+}$gives rise to the possibility of it being used as a marker to track the distribution of SAHx across a planetary surface, and a greater understanding of this phase's stability and thermal behavior could reveal much about the recent history of the icy crust it has formed upon.

\section{Conclusions}

[33] This work has identified a previously unknown sulfuric acid hydrate and, given the conditions of SAHx's formation, has demonstrated that it is likely to exist on the surfaces of the icy Galilean moons. The spectral properties of this new material should be distinct from other water-rich sulfuric acid hydrates. Its structure and physical properties may have a number of implications for the formation of surface and subsurface features upon the icy moons. Characterization of these basic building blocks of a planetary system is crucial information for the construction of more sophisticated models that will further our understanding of these worlds. With increasing evidence that Europa's surface is linked to its ocean below [Brown and Hand, 2013], the need for detailed understanding of its icy crust has become yet more apparent. The research presented here highlights the importance of detailed crystallographic investigations in the study of such materials. It is possible that "hexahydrate" could have been observed in earlier studies; however, without the collection of high-resolution powder diffraction, evidence of the existence of SAHx went undiscovered for nearly a century.

[34] Acknowledgments. Australian Synchrotron for the award of beamtime EPN 4785 on the powder diffraction beamline and the Australian Nuclear Science and Technology Organisation for the award of beamtime 2378 on the Echidna beamline, and AINSE for support of this beamtime. The authors wish to acknowledge Helen Brand of Australian Synchrotron and Norman Booth of the Bragg Institute both for technical assistance. HEMC acknowledges the Australian Synchrotron for postdoctoral funding.

\section{References}

Bergamaschi, A., A. Cervellino, R. Dinapoli, F. Gozzo, B. Henrich, I. Johnson, P. Kraft, A. Mozzanica, B. Schmitt, and X. T. Shi (2010), The MYTHEN detector for X-ray powder diffraction experiments at the Swiss Light Source, J. Synchrotron Radiat., 17, 653-668.

Boutonnet, A., and J. Schoenmaekers (2012), Mission analysis for the JUICE mission, in Spaceflight Mechanics 2012, edited by J. V. McAdams et al., pp. 1561-1578, Univelt Inc., San Diego.

Brand, H. E. A., A. D. Fortes, I. G. Wood, K. S. Knight, and L. Vocadlo (2009). The thermal expansion and crystal structure of mirabilite ( Na2SO4 center dot $10 \mathrm{D}(2) \mathrm{O}$ ) from 4.2 to $300 \mathrm{~K}$, determined by timeof-flight neutron powder diffraction, Phys. Chem. Miner., 36(1), 29-46.

Brown, M. E., and K. P. Hand (2013), Salts and radiation products on the surface of Europa, Astron. J., 145(4), 110.

Carlson, R. W., R. E. Johnson, and M. S. Anderson (1999), Sulfuric acid on Europa and the radiolytic sulfur cycle, Science, 286(5437), 97-99.

Carlson, R. W., M. S. Anderson, R. E. Johnson, M. B. Schulman, and A. H. Yavrouian (2002), Sulfuric acid production on Europa: The Radiolysis of sulfur in water ice, Icarus, 157(2), 456-463.

Carlson, R. W., M. S. Anderson, R. Mehlman, and R. E. Johnson (2005), Distribution of hydrate on Europa: Further evidence for sulfuric acid hydrate, Icarus, 177(2), 461-471

Cheng, A. F. (1984), Escape of sulfur and oxygen from Io, J. Geophys. Res., 89(A6), 3939-3944.

Coelho, A. (2008), TOPAS 4.1, Bruker AXS, Madison, Wisc. 
Cosier, J., and A. M. Glazer (1986), A nitrogen-gas-stream cryostat for general X-ray diffraction studies, J. Appl. Crystallogr., 19(2), $105-107$.

Dalton, J. B., D. P. Cruikshank, K. Stephan, T. B. McCord, A. Coustenis, R. W. Carlson, and A. Coradini (2010), Chemical Composition of Icy Satellite Surfaces, Space Sci. Rev., 153(1-4), 113-154.

Favre-Nicolin, V., and R. Cerny (2002), FOX, 'free objects for crystallography': A modular approach to ab initio structure determination from powder diffraction, J. Appl. Crystallogr., 35, 734-743.

Fortes, A. D., and M. Choukroun (2010), Phase Behaviour of Ices and Hydrates, Space Sci. Rev., 153(1-4), 185-218.

Fortes, A. D., I. G. Wood, and K. S. Knight (2006), Neutron powder diffraction studies of sulfuric acid hydrates. I. The structure of sulfuric acid hemitriskaidekahydrate D(2)SO(4)center dot $61 / 2 \mathrm{D}(2) \mathrm{O}$, J. Chem. Phys., 125(14), 144510.

Fortes, A. D., I. G. Wood, and K. S. Knight (2008a), The crystal structure and thermal expansion tensor of $\mathrm{MgSO}(4)-11 \mathrm{D}(2) \mathrm{O}$ (meridianiite) determined by neutron powder diffraction, Phys. Chem. Miner., 35(4), 207-221.

Fortes, A. D., I. G. Wood, L. Vocadlo, L. Chapon, K. S. Knight, and R. I. Smith (2008b), Neutron powder diffraction studies of sulfuric acid hydrates. II. The structure, thermal expansion, incompressibility, and polymorphism of sulfuric acid tetrahydrate $\left(\mathrm{D}_{2} \mathrm{SO}_{4} .4 \mathrm{D}_{2} \mathrm{O}\right), J$. Chem. Phys., 128(5), 054506.

Gable, C. M., H. F. Betz, and S. H. Maron (1950), Phase Equilibria of the System Sulfur Trioxide-Water1, J. Am. Chem. Soc., 72(4), $1445-1448$

Giran, H. (1913), Researches on sulfuric acid and sulfur trioxide, C. R. Hebd. Seances Acad. Sci., 157, 221-223.

Hirsch, T. K., and L. Ojamäe (2004), An investigation of H-atom positions in sulfuric acid crystal structures, Acta Crystallogr., Sect. B Struct. Sci., 60, 179-183.

Hornung, E. W., T. E. Brackett, and W. F. Giauque (1956), The Low Temperature Heat Capacity and Entropy of Sulfuric Acid Hemihexahydrate. Some Observations on Sulfuric Acid "Octahydrate"1, J. Am. Chem. Soc. $78(22), 5747-5751$

Hulsmann, O. (1934), The thermic analysis of the sulphuric acid/water system and the low temperature density of crystallised sulphuric acid hydrates, Z. Anorg. Allg. Chem., 218(4), 369-378.

Kjallman, T., and I. Olovsson (1972), Hydrogen-bond studies .58. Crystal structures of normat and deuterated sulfuric acid tetrahydrate $\left(\mathrm{H}_{5} \mathrm{O}_{2}\right)^{+2} \mathrm{SO}_{4}{ }^{2-}$ and $\left(\mathrm{D}_{5} \mathrm{O}_{2}\right)^{+2} \mathrm{SO}_{4}{ }^{2-}$, Acta Crystallogr., Sect. B Struct. Sci., 28(6), 1692-1697.
Kunzler, J. E., and W. F. Giauque (1952), The Heat Capacity and Entropy of Sulfuric Acid Trihydrate Glass and Crystals from 15 to $300^{\circ} \mathrm{K} .1, \mathrm{~J}$. Am. Chem. Soc., 74(3), 797-800.

Larson, A. C., and R. B. V. Dreele (1994), General structural analysis system (GSAS), Rep., Los Alamos National Laboratory.

Liss, K. D., B. Hunter, M. Hagen, T. Noakes, and S. Kennedy (2006), Echidna - the new high-resolution powder diffractometer being built at OPAL, Physica B, 385-86, 1010-1012.

Maynard-Casely, H. E., H. E. A. Brand, and K. S. Wallwork (2012), The structure and thermal expansion of sulfuric acid octahydrate, J. Appl. Crystallogr., 45, 1198-1207.

Mootz, D., and A. Merschenz-Quack (1987), Crystal Structure of acid hydrates and oxonium salts .28. On the highest hydrates of sulfruic acid - formation and structure of $\mathrm{H}_{2} \mathrm{SO}_{4} \cdot 6.5 \mathrm{H}_{2} \mathrm{O}$ and $\mathrm{H}_{2} \mathrm{SO}_{4} .8 \mathrm{H}_{2} \mathrm{O}, Z$ Naturforsch., B: J. Chem. Sci., 42(10), 1231-1236.

Peterson, S. W., and H. A. Levy (1957), A single crystal neutron diffraction study of heavy ice, Acta Crystallogr., 10(1), 70-76.

Pickering, S. U. (1890), XXV.-The nature of solutions, as elucidated by the freezing points of sulphuric acid solutions, J. Chem. Soc. Trans., 57, 331-369.

Prockter, L. M., R. M. C. Lopes, B. Giese, R. Jaumann, R. D. Lorenz, R. T. Pappalardo, G. W. Patterson, P. C. Thomas, E. P. Turtle, and R. J. Wagner (2010), Characteristics of Icy Surfaces, Space Sci. Rev. 153(1-4), 63-111.

Rottger, K., A. Endriss, J. Ihringer, S. Doyle, and W. F. Kuhs (2012) Lattice constants and thermal expansion of $\mathrm{H} 2 \mathrm{O}$ and D2O Ice Ih between 10 and 265 K (vol 50, pg 644, 1994), Acta Crystallogr., Sect. B Struct. Sci., 68, 91 .

Rubin, T. R., and W. F. Giauque (1952), The Heat Capacities and Entropies of Sulfuric Acid and Its Mono- and Dihydrates from 15 to $300^{\circ} \mathrm{K} .1, \mathrm{~J}$. Am. Chem. Soc., 74(3), 800-804.

Spek, A. (2009), Structure validation in chemical crystallography, Acta Crystallogr., Sect. D Biol. Crystallogr., 65(2), 148-155.

Spencer, J. R., L. K. Tamppari, T. Z. Martin, and L. D. Travis (1999), Temperatures on Europa from Galileo PhotopolarimeterRadiometer: Nighttime Thermal Anomalies, Science, 284(5419), 1514-1516

Wallwork, K. S., B. J. Kennedy, and D. Wang (2007), The high resolution powder diffraction beamline for the Australian Synchrotron, AIP Conf. Proc., 879, 879-882. 